\title{
Performance optimization of RSOA based mm-wave radio-over-fibre access network
}

\author{
Eszter Udvary ${ }^{1}$ (D)
}

Received: 12 February 2021 / Accepted: 20 June 2021 / Published online: 9 September 2021

(C) The Author(s) 2021

\begin{abstract}
In this paper, a Reflective Semiconductor Optical Amplifier based, Radio-over-Fibre access network configuration has been proposed to feed future millimeter-wave radio systems. The system architecture combines several approaches to overcome the challenges of millimeter-wave signal transmission. Reflective semiconductor optical amplifier modulator realizes a colorless and relatively cost-effective Remote Antenna Unit. The same optical carrier is used for both downlink and uplink. Optical single-sideband modulation is used at the downlink, which is robust against chromatic dispersion, but the complex realization of this modulation format is not possible at the Remote Antenna Unit. Optical intermediate frequency transmission is applied at the uplink direction, and the required local oscillator signal originates from the central station. The critical element is the reflective optical amplifier, as it compensates for the optical loss and works as an external intensity modulator. The operation of the reflective optical amplifier is modeled by multisection rate and wave equation-based description. The amplification and modulation behaviors of an available reflective optical amplifier are also measured. The experimental work validated the colorless operation and the quality of the modulation versus bias current and input optical power. Finally, system simulation was realized. The uplink and downlink power budgets were balanced, and optimal values for the optical coupling rate and RSOA bias current have been selected.
\end{abstract}

Keywords Optical access networks $\cdot$ Semiconductor optical amplifier $\cdot$ modulation

\section{Introduction}

The new generation of mobile communication networks requires a drastic rise in data speed. A potentially applicable method could be the higher spectral efficiency via millimeter-wave (mmWave) frequencies. The higher attenuation of the high-frequency radio signal transmission in free space requires smaller covered areas per radio access unit (RAU). Also, the high capacity per service area and the low power consumption operation require a

Eszter Udvary

udvary.eszter@vik.bme.hu

1 Department of Networked Systems and Services, Budapest University of Technology and Economics, Budapest, Hungary 
small cell size. The number of deployed RAUs must be increased to provide full coverage. A cost-effective RAU is necessary to meet these conditions. Radio-over-Fiber (RoF) technology (Novak et al. 2016) is a potentially applicable method. Analog RoF transmits the high-frequency, modulated radio frequency (RF) signals via relatively high distance optical fiber with tolerable quality degradation (Lim et al. 2014). In this approach, the complex functionalities and expensive devices, including the conversion between analog and digital ranges and signal processing, are moved to the Central Office (CO) (Gomes et al. 2016; Urban et al. 2016). Only the optical-electrical conversion and RF filtering and amplification remain in the RAUs. The full-duplex operation demands high-speed, colorless modulators at both $\mathrm{CO}$ and Rau. The operation of this device determines the link performance.

The semiconductor optical amplifiers allow for direct-modulated colorless transceivers in the system. It is a semiconductor-based, small size, low cost, current-driven device which requires low modulation power. The multifunctional capability of inline and reflective semiconductor optical amplifiers (SOAs and RSOAs) has already been demonstrated. The optical amplification can be combined with either modulation (Udvary 2003), photodetection (Udvary 2008), dispersion compensation (Udvary 2010a), linearization (Udvary $2010 \mathrm{~b}$ ), etc. The detected electrical power is high because of the optical gain of the SOAmodulator. Any other optical modulators have an optical insertion loss. It can be a crucial component for mmWave Radio over Fiber systems (mmWave-RoF, and new generation wavelength division multiplexed passive optical networks (WDM-PON).

Applying a reflective semiconductor optical amplifier as a modulator device in RoF systems has been suggested and investigated (Wake 2010). RSOA provides a cheaper option in numerous scenarios than Mach-Zehnder (MZ) modulator or directly modulated laser (DML). Furthermore, it meets the requirements of a wavelength-independent system (Medeiros et al. 2007). The limiting factors of the transmission quality must be considered, like nonlinear distortion, temperature-sensitive operation, and spontaneously emitted noise. In millimeter-wave applications, the modulation bandwidth could also become a significant limiting factor (Medeiros et al. 2007; Henning et al. 2016; Hailay et al. 2014). Modulation bandwidth can be enhancement thanks to the optical equalization effect of the chirping characteristics of RSOA (Udvary 2016; Rizou et al. 2020).

This paper investigates a specific RSOA based MMW Radio-over-Fiber transmission system by device measurements and simulations following system simulations. It determines the crucial points in the operation and gives general considerations for reaching better transmission quality. The system investigation focuses on the more critical uplink direction, but the results can be used for general considerations. The remainder of the paper is organized as follows. Section 2 presents the system concept of the investigated network architecture. Section 3 gives measurement results on the RSOA-modulator, which is the critical device in the system. Section 4 describes the simulation model of the RSOA-modulation function; it fits with the device measurements. Section 5 describes the simulation environment and provides system-level simulations and optimization results. Finally, the conclusion summarizes the paper.

\section{System concept}

The block scheme of the system concept can be seen in Fig. 1. A flexible optical access network was planned, applying cost-effective RAUs. Both downlink (DL) and uplink (UL) connections are realized in the $60 \mathrm{GHz}$ regime. The modulated DL subcarriers and the UL local 


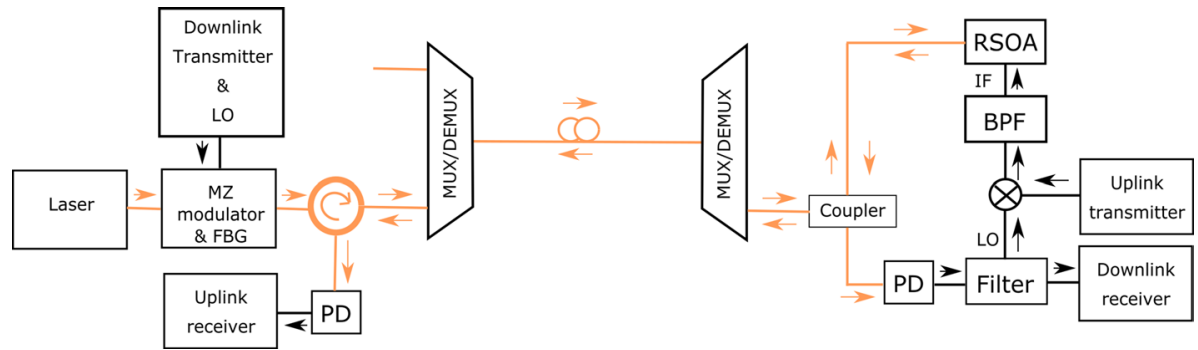

Fig. 1 Investigated network architecture. Laser: continous wave semiconductor laser source, LO: Local Oscillator, MZ Modulator: Mach-Zehnder modulator, PD: Photo diode, MUX: Multiplexer, DEMUX: demultiplexer, Filter: electrical branching filter, BPF: electrical BandPass Filter, RSOA: Reflective Semiconductor Optical Amplifier, coupler: optical power divider

oscillator (LO) signals intensity modulate the optical carrier in the $\mathrm{CO}$; a high-speed external MZ modulator is used. The incoming optical signal is split into two branches in the RAU. A high-speed photodetector converts one part of the transmitted optical signal to an electrical signal. Then the downlink receiver (DL Rx) processes the DL signal, and the transmitted LO signal is used for the downconversion in the mixer. The other part of the optical signal is directed to the RSOA, which amplifies and reflects the signal to the central office. The optical signal is intensity-modulated by the uplink information at the RSOA. The narrow RSOA modulation bandwidth limits the operation; the millimeter-wave subcarrier can not be applied. So, the uplink signals are down-converted to the intermediate frequency regime. The LO signal for downconversion is the same at all RAUs. It is transmitted from the CO via the access network; there are no separated LOs installed in all RAUs. The LO signal presents at the output of the photodetector in the RAU; then it is filtered and amplified and used as the LO input of the mixer.

The frequency dependence of the velocity in a transparent medium is called chromatic dispersion, the double-sideband (DSB) intensity-modulated optical signal consisting of the carrier and two sidebands. At the optical receiver, each sideband beats with the optical carrier; the RF signal is the sum of the beat signals. The chromatic dispersion causes a phase difference between the two components, which results in a power degradation of the composite RF signal. The chromatic dispersion caused phase difference depends on the modulation frequency, fiber length, and fiber dispersion parameter; as the RF frequency increases, the dispersion limits the transmission length at a shorter distance. The effect of chromatic dispersion is not negligible in millimeter-wave transmission. The degradation caused by dispersion can avoid applying Single Side Band (SSB) modulation; this approach was used at the DL. SSB can be realized by removing one sideband; it might be performed by filtering or using a unique modulation technique, like a dual-electrode Mach-Zehnder modulator. The applied RSOAmodulator provides DSB modulation. So, the DSB is applied at the UL, but the chromatic dispersion less affects the intermediate frequency than the millimeter-wave signals.

\section{Device measurements}

The operation of the RSOA-modulator device and the simulation results were experimentally verified. Figure 2 represents the simplified block diagram of the measurement setup. A tunable laser source produced the required optical power and wavelength. In some 
measurements, it was replaced by an optical multicarrier source. The continuous optical carrier is transmitted via optical fiber and directed to the optical connector of the RSOA. The RSOA-modulator under test was driven by the sum of bias current and sinusoidal modulation radio frequency signal applying a bias tee. A temperature control circuit eliminated the harmful effect of temperature dependence. The optical power at the output of the optical circulator is measured during the amplification tests. The detected electrical signal at the electrical output of the photodetector is registered during the modulation tests. A computer program controlled the setup; hence the measurement parameters were carefully set, and the measurement results were processed and stored.

In the first measurements, the amplification function was tested. A DWDM (Dense Wavelength Division Multiplexing) source was used with eight optical carriers. The RSOA amplified the eight optical channels parallel, and colorless operation in the $\mathrm{C}$ optical band was verified. Figure 3 shows the optical gain versus optical wavelength with different bias currents of the RSOA. The optical gain increases as the bias point increases, but the optical gain is about constant in the operation wavelength range.

In the next measurements, the optical source was changed to a tunable DWDM semiconductor laser. Figure 4 presents the Optical gain versus bias current curves in the case of different input optical powers. The optical gain is saturated as the input optical power increases. But all curves can be divided into three parts. In the first part, when the optical amplification effect starts, the curve is not linear. In the next part, the optical gain is linearly proportional to the bias current. Finally, the curve starts to saturate; this part of the curve is also nonlinear. The slope and boundary of the linear section depend on the input optical power.

In the second group of the measurements, the modulation behavior was tested by sinusoidal and baseband electrical signals. The bias circuit of the RSOA includes a bias tee, and the modulation content fluctuates the bias current. On the receiver side, a photodetector realizes the modulation information. First, baseband NRZ modulation format was used. The Q-factor characterizes the quality of the digital modulation content as a measure of the eye-opening; the optical gain is the ratio of the average optical powers at the ROSA output and input. During the tests, the bias current of the RSOA and the amplitude of the modulation content were changed; the optical power and the Q factor of the detected electrical signal were measured. The linear past of the RSOA gain-bias current curve was investigated. The optical gain and the quality of the transmitted modulation content versus the operation point of RSOA with different modulation depths can be seen in Fig. 5. The figure shows that all measured optical gain is on the linear regime. However, the different modulation amplitudes affected the quality of the information as the peak current reaches the nonlinear part of the curve. As the modulation

Fig. 2 Simplified block diagram of the RSOA-modulator characterization. Optical sourse: continous wave semiconductor laser source and external optical modulator, PD: Photo diode, RSOA: Reflective Semiconductor Optical Amplifier, DC: current source, OSA: Optical Spectrum Analyzer

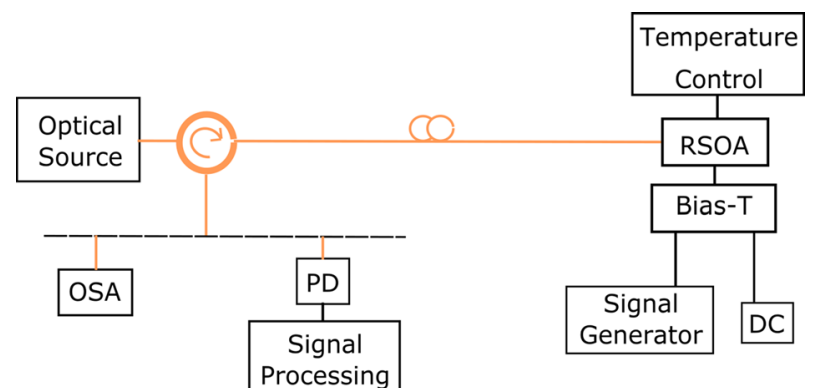




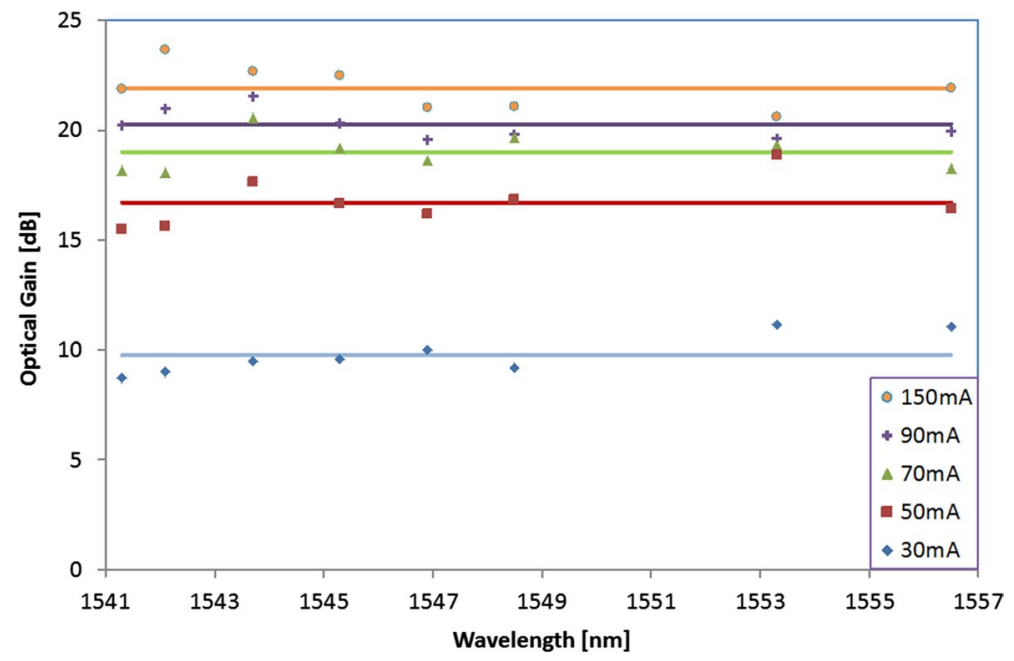

Fig. 3 Measured optical gain versus optical wavelength with different input optical powers

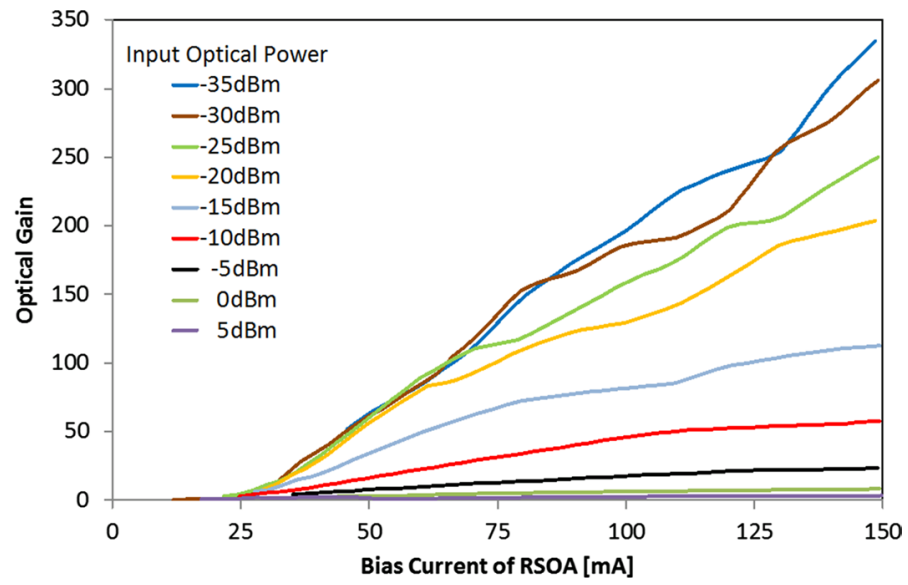

Fig. 4 Measured optical gain versus bias current of the RSOA with different input optical powers at $1550 \mathrm{~nm}$ optical wavelength

amplitude increases, the quality increases. But the higher modulation amplitude limits the usable bias current range.

Finally, the RF transfer function was measured. A sinusoidal RF signal generator was applied, the modulation frequency was swept, and a spectrum analyzer measured the power of the detected RF signal at the receiver side. The amplitude and shape of the transfer function mainly depend on input optical power and bias current. The transfer function is low pass; the bandwidth depends on the bias current of RSOA and the input optical power (Fig. 6). The improvement of the bandwidth is approximately directly proportional to the bias current. The slope of the curve is higher in the saturation regime, practically in higher input power. The modulation bandwidth is constant 


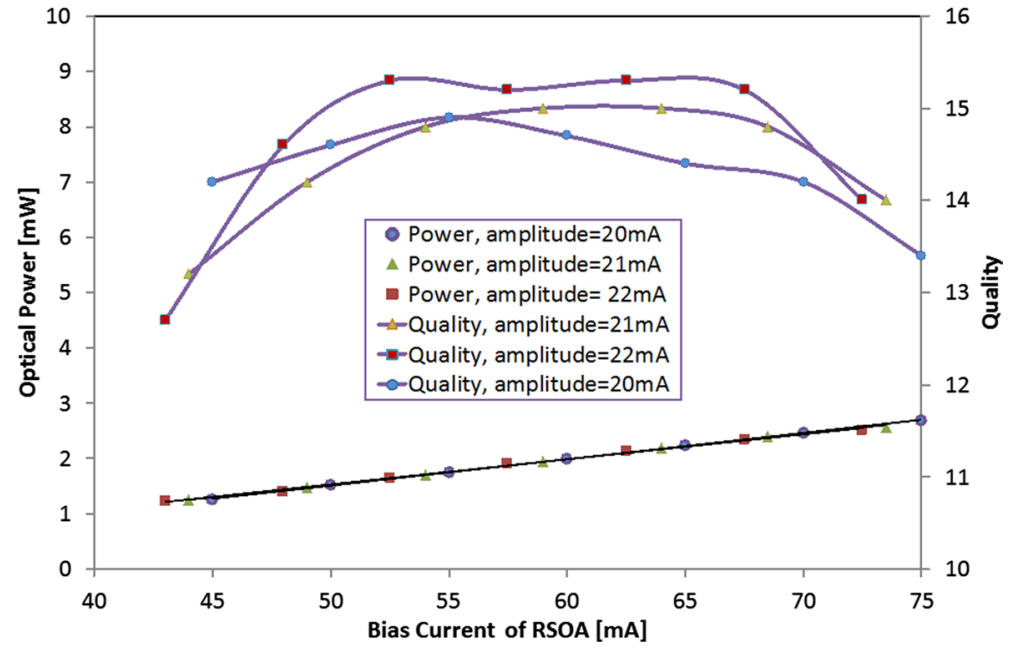

Fig. 5 Measured optical power and the quality of the transmitted electrical signal versus bias current of the RSOA

versus input optical power in the unsaturated regime, but it is improved in the saturated regime (Fig. 7).

\section{Device simulation}

The modulation functionality of the RSOA device was modeled based on the wave and rate equations. Material dynamic equations describe the time-dependent change in the semiconductor material that interacts with an optical field propagating via the RSOA. The rate equations describe the interaction of light with electrical carriers inside the RSOA. The

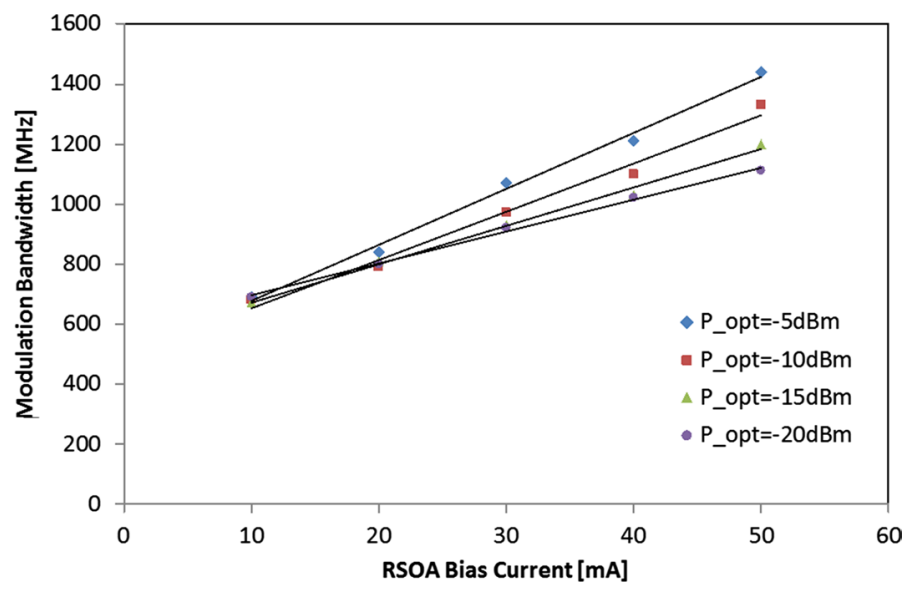

Fig. 6 Measured modulation bandwidth versus bias current of the RSOA 


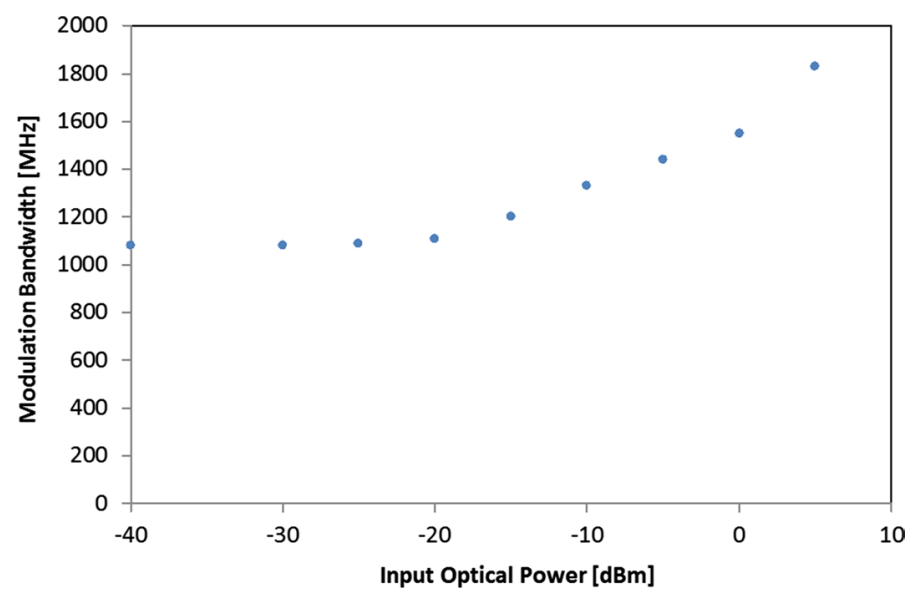

Fig. 7 Measured modulation bandwidth versus input optical power

spontaneous emission effect can extend the rate equations to describe the spontaneous emission caused saturation effect. The time and spatial-dependent rate equations are applied for photon density, and electrical carrier density (Udvary 2016)

$$
\begin{gathered}
\frac{\delta S(z, t)}{\delta z}=\Gamma \cdot a \cdot\left(N-N_{0}\right) \cdot S(z, t)-\alpha \cdot S(z, t) \\
\frac{\delta N(z, t)}{\delta t}=\frac{I(z, t)}{e \cdot V}-\frac{N}{\tau_{s}}-v_{g} \cdot a \cdot\left(N-N_{0}\right) \cdot S(z, t)
\end{gathered}
$$

where $S(z, t)$ is the photon density, $N(z, t)$ is the carrier density, $z$ is the position over the device length, $z=0$ is the input of the device, $z=L$ is the reflection point of the device, $t$ is the time, $v_{g}$ is the group velocity, $a$ is the differential gain, $\Gamma$ is the optical confinement factor, $N_{0}$ is the carrier density at transparency, $V$ is the volume of the active layer, $\tau_{s}$ is the lifetime of the electrical carrier, $\alpha$ is the internal loss (primarily waveguide scattering and absorption), $I$ is current, $e$ is the electron charge.

Similar equations can be defined for optical intensity and electrical current as the number of photons in the active layer gives the instantaneous optical intensity, i.e., the optical power.

$$
\begin{gathered}
I_{o p t}=h \cdot \frac{\omega_{o p t}}{2 \cdot \pi} \cdot v_{g} \cdot S \\
P_{o p t}=h \cdot \omega_{o p t} \cdot v_{g} \cdot A_{\text {mod }} \cdot S \\
A_{\text {mod }}=\frac{A}{\Gamma}
\end{gathered}
$$

where $A_{\text {mod }}$ is the mode area, $A$ is the geometric area, $\omega_{\text {opt }}$ is the circular optical frequency, $h$ is the Plank coefficient. And the material gain is the following.

$$
g(z, t)=\Gamma \cdot a \cdot\left(N-N_{0}\right)
$$


The input signal was assumed continuous (unmodulated), so the initial conditions at the input of the device include a constant component; the amplitude of the variable term is zero. The coupled rate and wave equations are solved numerically.

The carrier and photon densities are non-uniform along the inline SOA active region. This effect is less significant in RSOA, where the reflected optical signal balances the photon density, and the sorter device length decreases the spatial variation of the electrical carrier. Despite all these, the spatial variation can not be neglected; during the calculations, the location dependence was taken into account by dividing the device into longitudinal sections. Within a section, the spatial-dependent parameters are assumed to be constant. For each section, the average input and output photon densities are calculated one after the other. The sectioned amplifier cavity is used to account for longitudinal effects, and the spatial variation of the material gain and other parameters of the SOA can be captured.

After defining the model describing the operation of RSOA, similarly to describing the direct modulation of laser diodes, the current is divided into bias and radiofrequency modulation parts. Consequently, the carrier and photon densities also include constant and modulation parts. The operating point is defined by steady-state consideration. Next, the modulation part is calculated. The injected electrical signal change causes time-dependent carrier density, and the differential equation has to be applied. The modulation-associated change in the stimulated and spontaneous emissions can be calculated. The modulation amplitude of the photon density can be calculated the output of the device (Udvary 2016).

The small-signal amplitude is defined by the following formula, assuming sinusoidal modulation

$$
\frac{d \Delta S(z)}{d z}=\left(g_{\text {sat }}(z)-\alpha\right) \cdot \Delta S(z)+\Gamma \cdot a \cdot \Delta N(z) \cdot S_{D C}(z)
$$

where $S_{D C}$ is the average photon density, $g_{\text {sat }}$ is the saturated material gain, $S_{\text {sat }}$ is the saturation photon density, $g_{0}$ is the unsaturated material gain. The following equations define these parameters.

$$
\begin{gathered}
\frac{d S_{D C}}{d z}=\left(g_{s a t}-\alpha\right) \cdot S_{D C} \\
g_{s a t}=\frac{\Gamma \cdot g_{0}}{1+\frac{S_{D C}}{S_{s a t}}} \\
g_{0}=a \cdot\left(\frac{I_{D C}}{e \cdot V} \cdot \tau_{s}-N_{0}\right) \\
S_{s a t}=\frac{1}{a \cdot v_{g} \cdot \tau_{s}}=\frac{\Gamma \cdot P_{s a t}}{h \cdot \omega_{o p t} \cdot A \cdot v_{g}}
\end{gathered}
$$

Based on this derivation, the small-signal amplitude can be calculated using the following formula. 


$$
\begin{aligned}
\frac{d \Delta S(z)}{d z}= & \left(g_{s a t}(z)-\alpha\right) \cdot \Delta S(z) \\
& +\Gamma \cdot a \cdot \frac{\frac{\tau_{s}}{e \cdot V} \cdot \Delta I(z)-\frac{v_{g} \cdot \tau_{s} \cdot g_{s a t}(z)}{\Gamma} \cdot \Delta S(z)}{1+j \cdot \omega \cdot \tau_{s}+\frac{S_{D C}(z)}{S_{s a t}}} \cdot S_{D C}(z)
\end{aligned}
$$

The location dependence is also taken into account in the sectioned model when calculating the modulation content. Increasing the number of sections increases the accuracy of the model but also the runtime of the simulation.

The typically used electrode arrangement causes a significant propagation loss in the microwave frequency range $(3-30 \mathrm{GHz})$. The presented model is suitable for detecting the effect of the path taken by the modulating radio frequency signal to the device. The amplitude of the modulating signal must be set appropriately per section to test different electrode arrangements.

The presented description is suitable for detailed modeling of the operation of the device. It demonstrates the behavior of the gain function, such as optical gain saturation, operating current dependent optical gain, changes along propagation length, and optical noises. It also presents the modulation operation, including the dependence on the bias current, the modulation depth. The model also determines the modulation bandwidth. Different electrode layouts and multisection devices can be modeled with it (Manimaran 2020).

\section{System simulation}

The simulation and experimental investigation confirmed the effects of device and environmental parameters. Next, the system-level investigation is realized. The system was implemented in the Virtual Photonics Transmission Maker 9.0 simulation environment (VPI), the Matlab-based device model is integrated into the VPI environment. The simulation setup follows the optical access network architecture presented in Fig. 1.

A laser diode generates the continuous wave optical signal at $1550 \mathrm{~nm}$ with $10 \mathrm{dBm}$ optical power. The downlink information modulates the optical carrier by an external modulator. The modulator bias is set to the inflection point of the Mach-Zehnder modulator's sinusoidal transfer characteristic, which provides the most linear operation. The access network model consists of $10 \mathrm{~km}$ standard SMF28 fiber and multiplexer and demultiplexer for add and separate the optical carriers.

4 pieces of 16 QAM modulated subcarriers are applied with up to $325 \mathrm{Mbps}$ data speed in both uplink and downlink directions. The electrical subcarrier frequencies are in the $60 \mathrm{GHz}$ band with a $0.2 \mathrm{GHz}$ frequency distance for downlink direction (59.8, 60, 60.2, and $60.4 \mathrm{GHz}$ ). The $59 \mathrm{GHz}$ Local Oscillator (LO) signal for the uplink is transmitted parallel with the DL channels through the access network and recovered at the RAU. The optical signal is split with a $3 \mathrm{~dB}$ coupler (50\%) on the RAU side. One output is connected to the downlink receiver, the optical signal is detected, and the downlink channels are separated from the LO signal. The other output of the optical coupler is directed to the input of the RSOA biased by $75 \mathrm{~mA}$ bias current. The transmitted LO signal mixes down the UL $60 \mathrm{GHz}$ carriers to the intermediate frequency subcarriers in the $\mathrm{GHz}$ regime with $0.2 \mathrm{GHz}$ frequency distances $(0.8,1,1.2,1.4 \mathrm{GHz})$. The down-converted UL channels are added to the bias current of RSOA. The UL modulated optical signal is directed to the Central 
Table 1 Downlink symbol error rate for each subcarrier $(59.8 \mathrm{GHz}, 60 \mathrm{GHz}, 60.2 \mathrm{GHz}$, and $60.4 \mathrm{GHz}$ ) at three data rates (82 Mbps, 162 Mbps, 325 Mbps) and SSB modulation format at $325 \mathrm{Mbps}$

\begin{tabular}{lllll}
\hline & \multicolumn{2}{l}{ DSB } & & \multicolumn{1}{l}{ SSB } \\
\cline { 2 - 4 } & $82 \mathrm{Mbps}$ & $162 \mathrm{Mbps}$ & $325 \mathrm{Mbps}$ & $325 \mathrm{Mbps}$ \\
\hline $59.8 \mathrm{GHz}$ & $1.42 \times 10^{-5}$ & $8.69 \times 10^{-4}$ & $4.19 \times 10^{-2}$ & $1.88 \times 10^{-12}$ \\
$60.0 \mathrm{GHz}$ & $1.29 \times 10^{-7}$ & $1.93 \times 10^{-6}$ & $9.77 \times 10^{-4}$ & $2.04 \times 10^{-14}$ \\
$60.2 \mathrm{GHz}$ & $9.10 \times 10^{-8}$ & $2.62 \times 10^{-7}$ & $2.88 \times 10^{-5}$ & $6.10 \times 10^{-14}$ \\
$60.4 \mathrm{GHz}$ & $4.71 \times 10^{-12}$ & $3.64 \times 10^{-10}$ & $6.71 \times 10^{-7}$ & $1.07 \times 10^{-11}$ \\
\hline
\end{tabular}

\begin{tabular}{|c|c|c|c|c|}
\hline & \multicolumn{3}{|l|}{ DL DSB } & \multirow{2}{*}{$\begin{array}{l}\text { DL SSB } \\
325 \mathrm{Mbps}\end{array}$} \\
\hline & $82 \mathrm{Mbps}$ & $162 \mathrm{Mbps}$ & $325 \mathrm{Mbps}$ & \\
\hline $0.8 \mathrm{GHz}$ & $2.64 \times 10^{-8}$ & $5.73 \times 10^{-8}$ & $6.95 \times 10^{-6}$ & $2.41 \times 10^{-6}$ \\
\hline $1.0 \mathrm{GHz}$ & $7.66 \times 10^{-8}$ & $8.44 \times 10^{-8}$ & $1.44 \times 10^{-5}$ & $3.91 \times 10^{-5}$ \\
\hline $1.2 \mathrm{GHz}$ & $1.16 \times 10^{-6}$ & $3.53 \times 10^{-6}$ & $1.34 \times 10^{-4}$ & $2.80 \times 10^{-4}$ \\
\hline $1.4 \mathrm{GHz}$ & $1.31 \times 10^{-5}$ & $3.12 \times 10^{-5}$ & $3.20 \times 10^{-4}$ & $1.03 \times 10^{-3}$ \\
\hline
\end{tabular}

Table 2 Uplink symbol error rate for each subcarrier $(0.8 \mathrm{GHz}$, $1 \mathrm{GHz}, 1.2 \mathrm{GHz}, 1.4 \mathrm{GHz})$ at three data rates $(82 \mathrm{Mbps}$, $162 \mathrm{Mbps}, 325 \mathrm{Mbps}$ ) and the effect of the downlink SSB modulation form $325 \mathrm{Mbps}$ uplink data speed

Office, where the optical circulator separates the two directions, the UL signal is detected and processed.

The symbol error rate (SER) qualifies the data transmission. First, the DSB intensity modulation was tested at the DL. Table 1 . shows that the transmission quality is sensitive for the subcarrier frequency. The SER of the transmitted $325 \mathrm{Mbps}$ datastream is about 0.05 at $59.8 \mathrm{GHz}$, and it decreases to $10^{-6}$ as the subcarrier frequency rises to $60.4 \mathrm{GHz}$. The reason is the chromatic dispersion induced high attenuation at the $59.8 \mathrm{GHz}$ frequency. The SER is better at lower data speed, but the same subcarrier dependence can be observed. The effect can be eliminated by optimizing subcarrier frequencies and link length, as the chromatic dispersion caused deep in the transfer function is narrow and fiber length-dependent, but these methods are not usually practically applicable.

In the next case, optical SSB modulation was tested by applying Fiber Bragg Grating (FBG) filtering. The used FBG has a $13 \mathrm{GHz}$ wide stopband and $30 \mathrm{~dB}$ suppression. The SSB robust again the chromatic dispersion, it is validated by the simulation results, also presented in Table 1. Despite the attenuation of the FBG due to the elimination of one sideband, the transmission quality is better and more balanced over the frequency.

The UL transmission was also tested at different data speeds (Table 2). The UL quality is not affected by the chromatic dispersion effect because of the lower frequency range. The modulation bandwidth of the RSOA causes worse quality at higher frequencies. DSB modulation format is used for the uplink as the chromatic dispersion does not degrade the transmission. However, the SSB applied at the DL changes the level of the UL LO signal. The UL's quality is a little degraded by the SSB modulation at the DL; it is presented in the last coulomb in Table 2.

However, the uplink transmission operates with significantly more symbol errors than the downlink direction. The average downlink SER is below $10^{-10}$; the uplink SER is about $10^{-4}$. According to these results, the RSOA operates as a less reliable modulator than the Mach-Zehnder.

The operation of the RSOA is colorless; it can be applied in a flexible, dynamically reconfigurable system. The change of wavelength alters the gain and consequently the 
modulation characteristic of the device. This effect is deterministic, determined by the used wavelengths. The temperature dependence can also change the transmission quality (Straullu 2012).

As the qualities of UL and DL are unbalanced, two adjustments are proposed and verified to reach lower SER values in the uplink direction.

\subsection{Coupling ratio adjustment}

The modulation behavior of the RSOA depends on the input optical power due to the saturation effect. The link's power budget can be modified without changing the laser power with the optical coupler ratio adjustment. This suggested method does not require extra cost because optical couplers with different rates can be widely found on the market.

The coupler modification can positively affect the UL transmission quality, but several phenomenons need to be considered. The decreased optical power reaches the receiver photodiode, resulting in a higher downlink symbol error rate due to the SNR reduction. Also, the local oscillator power decreases as it is sen parallel with the DL channels. It causes a noisier LO signal of the mixer degrading the UL. The input optical power of RSOA affects the amplification characteristics due to the saturation effect, the output optical power changes, and the optical power budget is affected. Higher optical input power increases the RSOA modulation bandwidth because of the saturation effect. Higher optical input power decreases the amplified spontaneous emission power due to the saturation effect, and the noise level is decreased. The modulation depth is changed due to decreased LO power and a more saturated optical amplifier.

The coupler modification effect was simulated from 70/30 to $1 / 99 \%$ coupling ratio, the uplink SER versus coupling rate can be seen in Fig. 8. As higher optical power reaches the input of the RSOA, an optimal point can be realized with a minimum symbol error rate. This point is the $20 / 80 \%$ coupler rate in our setup, but the exact value depends on the actual parameters of the system. With this setting, the average uplink symbol error rate is in the range of $10^{-6}$, which is a significant improvement compared to the initial $50 / 50 \%$ ratio. Same time, the downlink SER is still sufficiently low; it is about $10^{-11}$ on average.

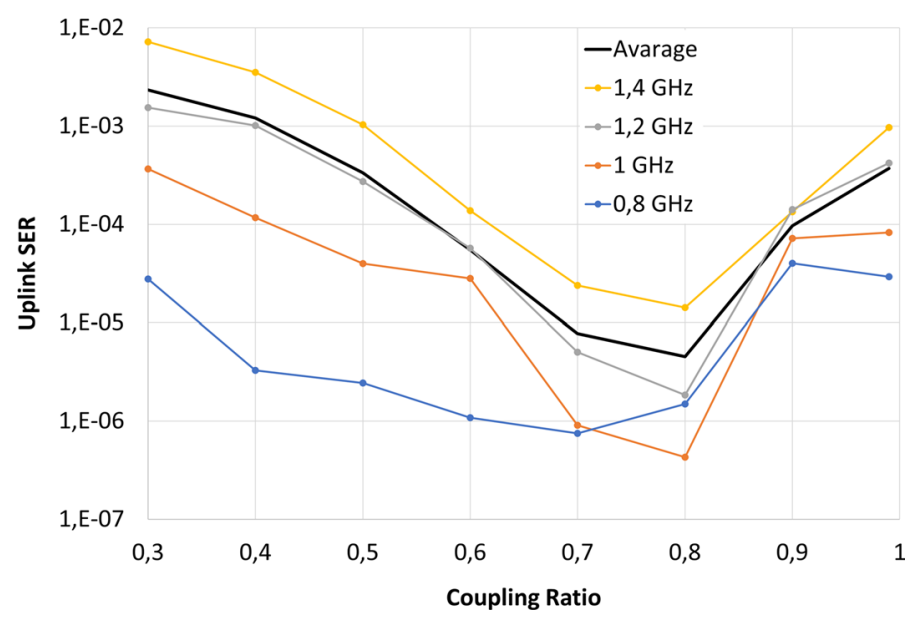

Fig. 8 System simulation results, effect of coupler tuning on uplink symbol error rate 
The results show that shifting away from the 50/50\% regular ratio, a more optimal setting can be found to minimize the uplink symbol error rate. However, precise fine-tuning is required in every specific case.

\subsection{RSOA bias adjustment}

The power budget of the system also depends on the RSOA bias, but it only affects the uplink error symbol rate. As the bias current of ROA increases, the optical gain and the modulation bandwidth increase. The higher optical gain causes higher optical output power, and the UL power budget is improved. The extended modulation bandwidth improves the modulation characteristics of ROSA. The combined effect of the two phenomena is shown in Fig. 9. Consequently, the high bias current positively affects the modulation parameters overall and improves transmission quality. However, higher bias values increase the power consumption on the receiver side, which could be a significant drawback in some applications and also can reduce the RSOA lifetime.

\section{Conclusion}

An RSOA-based, colorless, Radio-over-Fibre access network configuration has been proposed to feed future millimeter-wave radio systems. The multisection rate and wave equation-based RSOA model describes amplification and modulation behaviors validated by measurement. The transmission quality of the system was investigated by simulation. Based on the results, the transmission quality in the uplink direction is significantly lower compared to the downlink. Two methods are proposed to achieve more reliable uplink communication. The system simulation results validated the efficiency of the suggested methods. The coupling ratio adjustment allowed us to decrease the uplink symbol error rate from $10^{-4}$ down to $10^{-6}$, while the downlink SER stayed low. The advantages and also disadvantages of the approaches are presented.

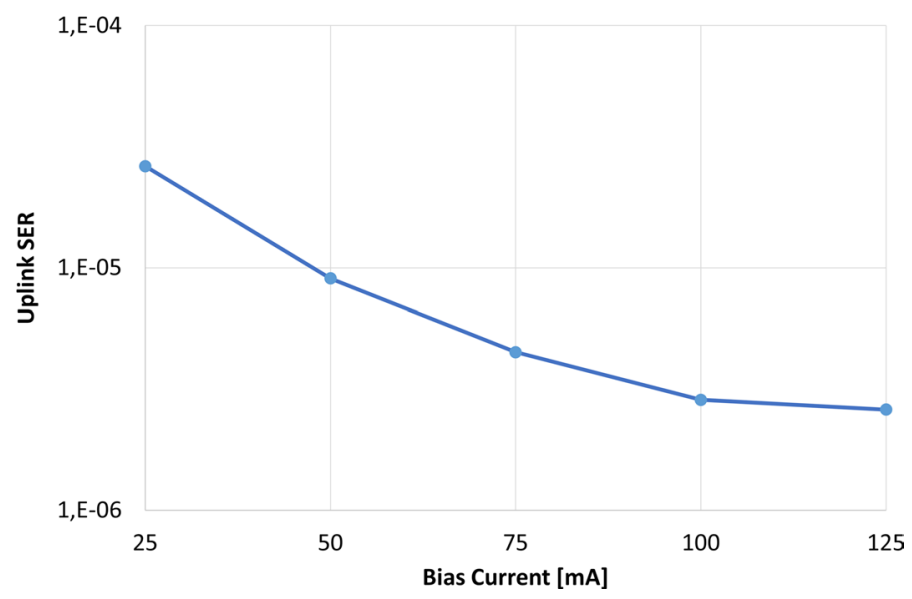

Fig. 9 System simulation results: effect of ROSA bias current tuning on uplink symbol error rate, $325 \mathrm{Mbps}$ 
Acknowledgements The author thanks Mr. David Kobor for his valuable contribution to the system simulation in VPI environment. This research was supported by the Ministry of Innovation and Technology and the National Research, Development and Innovation Office within the Quantum Information National Laboratory of Hungary.

Funding Open access funding provided by Budapest University of Technology and Economics.

Availability of data and materials All data generated or analysed during this study are included in this published article.

\section{Declarations}

Conflict of interest The author declare that he has no conflict of interest.

Open Access This article is licensed under a Creative Commons Attribution 4.0 International License, which permits use, sharing, adaptation, distribution and reproduction in any medium or format, as long as you give appropriate credit to the original author(s) and the source, provide a link to the Creative Commons licence, and indicate if changes were made. The images or other third party material in this article are included in the article's Creative Commons licence, unless indicated otherwise in a credit line to the material. If material is not included in the article's Creative Commons licence and your intended use is not permitted by statutory regulation or exceeds the permitted use, you will need to obtain permission directly from the copyright holder. To view a copy of this licence, visit http://creativecommons.org/licenses/by/4.0/.

\section{References}

Gomes, N.J. et al.: The new flexible mobile fronthaul: digital or analog, or both? In: International Conference on Transparent Optical Networks (2016)

Hailay, A. et al.: RoF using optically equalized RSOA WDM-PON architecture. In: International Conference on Transparent Optical Networks (2014)

Henning, L.F., et al.: High spectral wavelength agnostic multicarrier D-RoF modem based on uncooled RSOA. IEEE Photonics Technol. Lett. 28(9), 1010-1013 (2016)

Lim, C., et al.: Wireless signals transport in fiber-wireless links: digitized versus analog. In: 12th International Conference on Optical Internet Proceedings (2014)

Manimaran, P. et al.: Bandwidth characteristics of TWSOA based inline detector configurations for near infrared communications. Opt. Quantum Electron. 52, 1-6 (2020)

Medeiros, M.C.R., et al.: Radio over fiber access network architecture employing reflective semiconductor optical amplifiers. In: International Conference on Transparent Optical Networks, Mediterranean Winter, Sousse, Tunisia, December 6-8 (2007)

Novak, D., et al.: Radio-over-fiber technologies for emerging wireless systems. IEEE J. Quantum Electron. 52, 1-11 (2016)

Rizou, Z.V., et al.: Reflective semiconductor optical amplifier direct modulation capability enhancement using birefringent fiber loop. Appl. Sci. (2020). https://doi.org/10.3390/app10155328

Straullu, S., et al.: Characterization of uncooled RSOA for upstream transmission in WDM reflective PONs. Opt. Express 20(26), B38-B44 (2012)

Udvary, E., et al.: Optical subcarrier label swapping by semiconductor optical amplifiers. IEEE J. Lightwave Technol. 21(12), 3221-3225 (2003)

Udvary, E., et al.: Semiconductor optical amplifier for detection function in radio over fiber systems. IEEE J. Lightwave Technol. 26(8), 2563-2570 (2008)

Udvary, E., et al.: Combined optical amplification and dispersion compensation in radio over fibre link. IET Optoelectron. 4(6), 260-266 (2010)

Udvary, E., et al.: Improvements in the linearity of semiconductor optical amplifiers as external modulators. IEEE Trans. Microwave Theory Techn. 58(11), 3161-6167 (2010)

Udvary, E.: Off-set filtering effect in SOA based optical access network. Radioengineering 25(1), 26-33 (2016)

Urban, P.J., et al.: Fiber monitoring using a sub-carrier band in a sub-carrier multiplexed radio-over-fiber transmission system for applications in analog mobile fronthaul. J. Lightwave Technol. 34(13), 31183125 (2016) 
Wake, D., et al.: RA comparison of radio over fiber link types for the support of wideband radio channels. J. Lightwave Technol. 28(16), 2416-2422 (2010)

Publisher's Note Springer Nature remains neutral with regard to jurisdictional claims in published maps and institutional affiliations. 